\title{
Personalised Adaptive CBR Bolus Recommender System for Type 1 Diabetes
}

\author{
Ferran Torrent-Fontbona and Beatriz López
}

\begin{abstract}
Type 1 Diabetes Mellitus (T1DM) is a chronic disease. Those who have it must administer themselves with insulin to control their blood glucose level. It is difficult to estimate the correct insulin dosage due to the complex glucose metabolism, which can lead to less than optimal blood glucose levels. This paper presents PepperRec, a case-based reasoning (CBR) bolus insulin recommender system capable of dealing with an unrestricted number of situations in which T1DM persons can find themselves. PepperRec considers several factors that affect glucose metabolism, such as data about the physical activity of the user, and can also cope with missing values for these factors. Based on CBR methodology, PepperRec uses new methods to adapt past recommendations to the current state of the user, and retains updated historical patient information to deal with slow and gradual changes in the patient over time (concept drift). The proposed approach is tested using the UVA/PADOVA simulator with 33 virtual subjects and compared with other methods in the literature, and with the default insulin therapy of the simulator. The achieved results demonstrate that PepperRec increases the amount of time the users are in their target glycaemic range, reduces the time spent below it, while maintaining, or even reducing, the time spent above it.
\end{abstract}

Index Terms-Diabetes, bolus recommender system, case based reasoning, patient empowerment.

\section{INTRODUCTION}

$\mathbf{T}$ YPE 1 Diabetes Mellitus (T1DM) is a chronic metabolic disease characterised by the autoimmune destruction of the beta cells of the endocrine pancreas responsible for controlling Blood Glucose (BG) levels through the secretion of insulin. There are no actual global statistics regarding the number of people suffering from T1DM [1], but the American Diabetes Association estimates that there are approximately 1.25 million American adults and children with T1DM. According to the International Diabetes Federation, the highest incidence rates are in Europe, where there are approximately 140,000 children with T1DM and 21,600 new cases every year [2].

People with T1DM must control their BG level, e.g. by drawing blood from their fingertips in order to measure the glucose concentration, and then administer insulin to mimic the natural insulin secretion of the pancreatic beta cells. Large intervention trials, [3], have shown the impact of tight glycaemic control to avoid hyperglycaemia, and its consequent long term micro-vascular (retinopathy, nephropathy and neuropathy) and macro-vascular (coronary heart disease, stroke and peripheral vascular disease) complications. These trials

F. Torrent-Fontbona and B. López are with eXiT research group of Institute of Informatics and Applications, University of Girona, Girona, email: ferran.torrent@udg.edu

Manuscript received July DD, 2017; revised MMMM DD, YYYY. have highlighted the need for accurate and timely insulin dosage to avoid hypoglycaemia.

In practice, people with T1DM combine basal insulin (slowacting insulin) and bolus insulin (fast-acting insulin) to control BG. Basal insulin is adjusted by clinicians in collaboration with patients during periodic visits. Bolus dosage parameters, the Insulin to Carbohydrates Ratio (ICR) and the Insulin Sensitivity Factor (ISF), are also adjusted by clinicians in collaboration with patients, but bolus doses must be calculated by patients each time they are required which is usually before an intake of carbohydrates. Bolus doses are calculated according to the agreed ICR, ISF, carbohydrate intake, the blood glucose level and the active insulin remaining from previous doses (insulin on board). The final bolus may be adjusted by the user, depending on other factors such as the time of day or their physical activity. There are several insulin bolus calculators on the market to help people calculate their insulin dosage [4]. These are software tools usually incorporated in insulin pumps, glucose meters, personal digital assistants and smartphones, such as [5] and [6].

Bolus calculators have proved useful in improving patients' glycaemic self-control [7], [8], [9], [10]. However, they do not achieve optimal glycaemic control, usually because of the difficulty associated with setting parameters and regularly adjusting them to take account of changes in the patients' insulin requirements due to their physical activity, time of day, etc. These decisions are taken according to simple rules based on empirical experience. However, these simple rules do not capture the complexity of the glucose metabolism. This leads to miscalculations with regard to the appropriate insulin dosage. Therefore, there is a need to develop insulin recommender systems that aid people with T1DM.

Case Based Reasoning (CBR) [11] is a methodology that provides new recommendations based on past ones. This technique is becoming an alternative to building tools to support users' decisions. In this paper, we present PepperRec, a novel approach developed as part of the H2020 project PEPPER, for insulin bolus recommendations based on CBR that focuses on the management of an unrestricted number of situations (cases) in which the users find themselves. These situations depend on a set of variables (time of day, meal size, and past and future physical activity), some of which could have missing values. PepperRec estimates the ICR and ISF according to the situation of the user, and then calculates the bolus recommendation using these parameters. PepperRec can manage patient physiological evolution and model the slow changes of the user over time (concept drift [12]).

CBR for insulin recommender systems was first proposed 
in [13], but it achieved limited results in practice due to the technological limits and capabilities of the mobile devices at the time. Brown extended this work in [14] and proposed a bolus recommender system based on CBR that runs in a mobile application. It consists of a CBR system which considers the $\mathrm{BG}$, time and carbohydrate intake of several previous meals in order to recommend a bolus dose. In contrast to PepperRec, the method in [14] provides the bolus dose, while PepperRec calculates the ICR and ISF parameters from which the bolus is obtained. Therefore, [14] needs more cases to cover the possible combinations of the input variables, whereas PepperRec estimates the ICR, ISF and then calculates the bolus using them, the carbohydrate intake and the BG level, which requires a smaller number of cases.

Herrero et al. in [15], [16] proposed two approaches based on CBR to deal with different contexts. Three possible times of day and three physical activity levels define up to nine possible contexts with the corresponding ICR and ISF values. When a bolus recommendation is required, the system evaluates the current situation (time of day and exercise) and then uses the ICR and ISF that correspond to the most similar situation stored to calculate the required bolus. The methodology then checks the output of the proposed bolus dose, in the form of the postprandial blood glucose level, and corrects the used ICR and ISF using a run-to-run algorithm (R2R). Therefore, the methods proposed in [15], [16] are capable of repeatedly adjusting the parameters of a bolus calculator (ICR and ISF) for different contexts. Similarly to [15], [16], this paper proposes a method based on CBR that automatically adjusts the ICR and ISF, and then calculates the required bolus for a given carbohydrate intake and BG level. However, [15], [16] copy the solution from past experiences and then revise them, whereas PepperRec provides a reuse method to adapt past ICR and ISF values to the current situation, as well as a new revise and retain mechanism.

In addition to CBR, other artificial intelligence techniques have been explored for bolus recommendation. For example, [17] proposes a bolus recommender system based on fuzzy logic. It identifies a physiological model for the corresponding user using information about how the ICR changes depending on meal size. Then the fuzzy logic model is repeatedly updated after each meal to adjust the recommendations to the user's physiology. However, this model only catches ICR variability caused by the amount of carbohydrates in the meal. Authors in [18] propose a R2R method that repeatedly adjusts user ICR and ISF and then calculates the appropriate bolus dose for a given BG level and carbohydrate intake. This is a method that is capable of personalising a subject's ICR and ISF, and capturing possible changes over time (inter-day variability). However, ICR and ISF depend on several factors and do not usually have a monotonic evolution. Rather, they have periodicities due to intra-day variability, peaks and valleys due to non-constant physical activity, stress, hormone cycle, etc. Therefore, CBR alternatives, such as PepperRec have been developed as more powerful tools in terms of capturing this variability.

Other algorithms to calculate the required insulin dosage are proposed for closed-loop BG control systems, such as

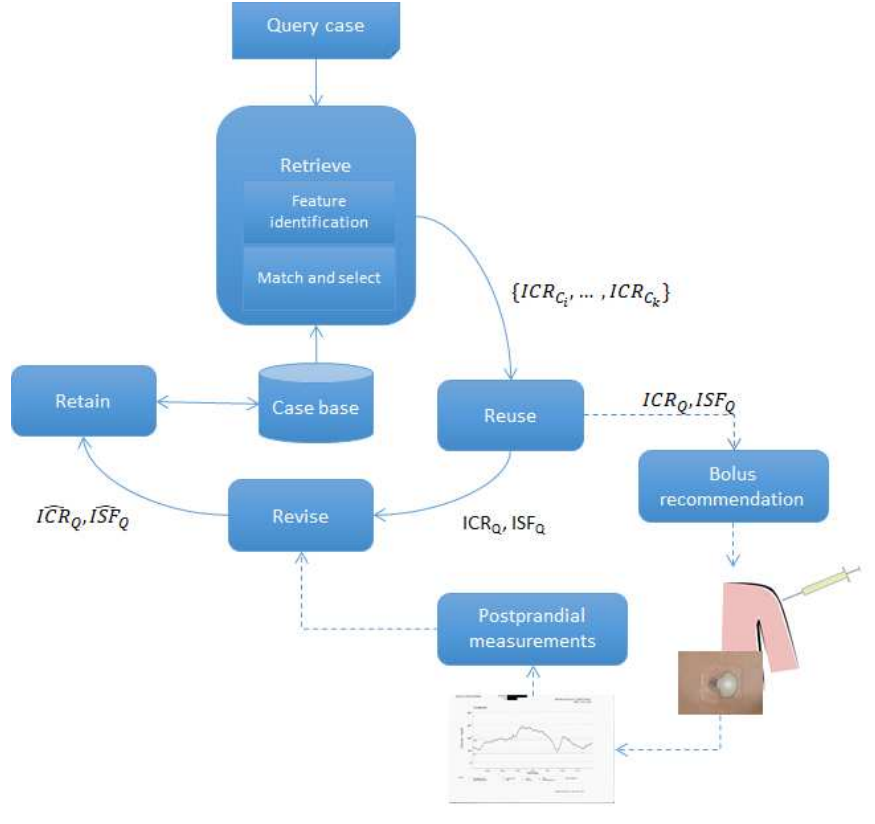

Fig. 1. PepperRec approach.

the ones suggested in [19], [20] or those reviewed in [7], [21]. However, these algorithms are usually control algorithms that use glucose predictions to regulate a continuous (or almost continuous) infusion of insulin. Conversely, this paper proposes a method for recommending insulin doses for people with T1DM, where the user decides whether or not to accept the outcome. Therefore, closed-loop algorithms are not applicable.

\section{Materials AND Methods}

PepperRec is a personalised and adaptive CBR bolus recommender system for people with T1DM. Those using this recommender system are assumed to wear a Continuous Glucose Monitor (CGM) to monitor BG, especially during the postprandial phase. The proposed bolus recommender system first estimates the ICR, and then calculates the ISF. The ICR and ISF are parameters whose setting is one of the main difficulties that clinicians, carers and people with diabetes face when they use bolus calculators [15]. Once the ICR and ISF are estimated, PepperRec calculates the bolus.

Figure 1 illustrates PepperRec methodology for bolus recommendation. The details are described in the reminder of the section.

\section{A. $C B R$}

CBR combines problem solving and lazy learning techniques to work out a solution for a new problem based on past experience [11]. Given a problem or query case Q, this methodology obtains a solution by following four main phases: (i) retrieve of past experiences similar to $\mathrm{Q}$, (ii) reuse of the retrieved past solutions to work out a new solution for the new problem, (iii) revision of the proposed solution according to the outcome, and (iv) retention of the new case according to 


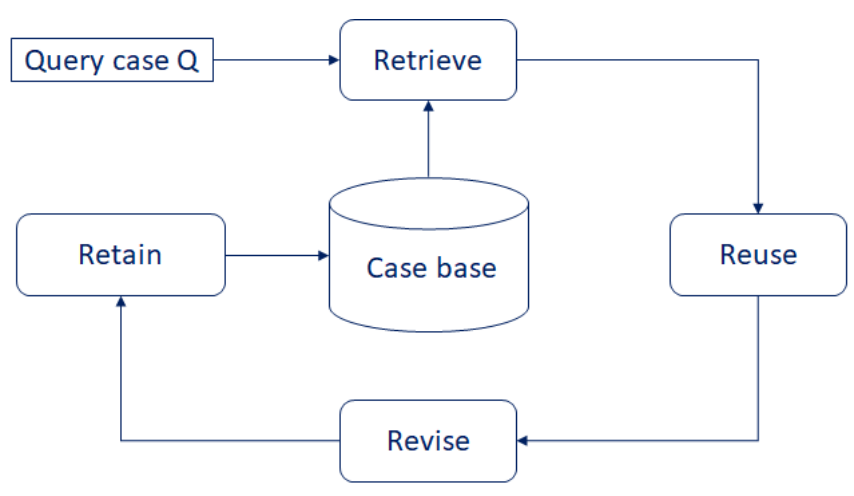

Fig. 2. CBR cycle.

TABLE I

CASE REPRESENTATION

\begin{tabular}{|c|c|c|}
\hline Type & Variable & Description \\
\hline Input (retrieve) & Time of day & Hour of the day \\
Input (retrieve) & $C H O$ & Amount of carbohydrates of the meal (in $g$ ) \\
Input (retrieve) & Past physical activity & Four-level quantification of past exercise \\
Input (retrieve) & Future physical activity & Four-level estimation of future exercise \\
\hline Input (reuse) & $G_{c}$ & Preprandial blood glucose (in $m g / d l$ ) \\
Input (reuse) & $I O B$ & Insulin on board \\
Output (reuse) & $I C R_{Q}$ & Estimated ICR for the current case \\
Output (reuse) & $I S F_{Q}$ & Estimated ISF for the current case \\
\hline Input (revise) & $W$ & Subject's weight (in $\mathrm{kg}$ ) \\
Input (revise) & $G_{s p}$ & Target blood glucose (in $\mathrm{mg} / \mathrm{dl}$ ) \\
Input (revise) & $G_{l}$ & Lower bound blood glucose (in $\mathrm{mg} / \mathrm{dl}$ ) \\
Input (revise) & $G_{\min }$ & Postprandial minimum glucose (in $\mathrm{mg} / \mathrm{dl}$ ) \\
Output (revise) & $I C \hat{R}_{Q}$ & Corrected ICR \\
Output (revise) & $I S F_{Q}$ & Corrected ISF \\
\hline
\end{tabular}

a strategy that considers its relevance measured according to certain metrics.

Figure 2 illustrates the CBR methodology.

The core of the methodology is the case base, where experiences are stored. In the case of insulin recommendation, as cases capture the history of the person's insulin management, the outcome of PepperRec is a personalised recommendation. Therefore, the size and quality of the cases stored determines the quality of the CBR outcomes. Retain mechanisms are crucial for this purpose, and can be complemented with other additional maintenance steps as proposed in [22].

\section{B. Cases}

The structure of the cases stored in the case base is shown in Table I. The input variables are the time of day, the estimated carbohydrate intake, the current BG level, and past and future physical activity. Amongst these parameters, physical activity has been identified as a key factor in this work. Therefore, a particular descriptor, which consists of a four-level quantification, is inferred in the retrieve step for physical activity.

\section{Retrieve}

The retrieve step selects similar cases to the query (or new) case from the input data. Two main steps are considered in this phase: feature identification, and the match-and-selection of cases.
1) Feature identification: In the feature identification step, several inferences are made regarding the input variables. Physical activity before a meal is quantified in four levels according to the exercise performed in the last hours measured by an activity tracker. If its use is consistent, the activity tracker ensures an objective and consistent measurement of the activity. However, this does not produce accurate measurements due to the quality of the activity tracker sensors. Nevertheless, as CBR can follow up a patient's subjective information, it could do the same with the information captured by the activity tracker [22].

The four quantification levels (none, low, mild and intense) are defined with personal thresholds:

- None: activity below th1

- Low: activity between $t h 1$ and $t h 2$

- Mild: activity between $t h 2$ and $t h 3$

- Intense: activity over $t h 3$

where $t h 1, t h 2$ and $t h 3$ are thresholds which depend on the average activity of the user during this period. The user is also asked to provide an approximate four-level (none, low, mild, intense) subjective prediction of the physical activity they will carry out during the postprandial phase.

The size of the meal (CHO) also has an impact on the ICR and ISF. Therefore, the amount of carbohydrates is quantified on three levels (low, medium, high) depending on whether the meal has less than $30 \mathrm{~g}$, more than $30 \mathrm{~g}$ and less than $70 \mathrm{~g}$, or more than $70 \mathrm{~g}$ of carbohydrates, respectively. The variable indicating the hour of the day is already a discrete variable since its possible values are the integer hours of the day, i.e. from 0 to 23. Therefore, the hour of the day is adjusted using a rounding procedure based on the timestamp.

Once all the variables used in the retrieve step are quantified, they are normalised to values in $[0,1]$. Note that $B G$ is not used by the retrieve step and therefore it is not quantified or normalised.

2) Match and select: The retrieval of similar experiences is based on a similarity measure. When choosing the appropriate similarity measure, it is important to consider that in healthcare and medicine, the available data is rarely complete. Therefore, systems should be able to deal with missing values [23], [24].

The proposed similarity measure is inspired in Tversky index [25] and consists of an average Euclidean distance between all the attributes, but with the particularity that it is normalised by the number of non-missing values of the query case. Thus, given a query case $Q$ with $\left\{N_{Q}\right\}$ non-missing attributes, and a case $C$ from the case base, then the similarity between the two cases is given by the Equation (1), where $N$ is the number of attributes, and $q_{i}$ and $c_{i}$ are the $i$ th attribute of case $Q$ and case $C$, respectively. Moreover, the distance between a non-missing value and a missing value is considered maximum, i.e. 1 .

$$
S(Q, C)=1-\frac{1}{\|\left\{N_{Q} \|\right\}} \sum_{i \in\left\{N_{Q}\right\}}\left(q_{i}-c_{i}\right)^{2}
$$

Note, that the proposed similarity measure does not fulfil that $S(Q, C)=S(C, Q)$ if both cases have different missing attributes. However, this ensures that the selected cases are 
those more similar to the query case considering the known information of the query case. Other techniques such as replacing the unknown value with an average value may introduce bias in the selection of cases. Forcing the distance between a known attribute of the query case and an unknown attribute of a case from the case base as 1, leads the retrieve step to prefer the most complete cases (those with more known attributes) in the case base. The outcome of the retrieval stage is a set of the $K$ most similar cases.

\section{Reuse}

As the retrieved and query situations are not identical, PepperRec provides the ICR solution to the query case following [26]. The proposed reuse methodology consists of a weighted mean of the ICR of the retrieved cases based on their similarity to the query case as shown in Equation (2), where $K$ is the number of retrieved cases, $I C R_{Q}$ is the ICR of the query case, $C_{k}$ is the $k$ th retrieved case, and $I C R_{C_{k}}$ is its corresponding ICR.

$$
I C R_{Q}=\frac{\sum_{i=1}^{K} S\left(Q, C_{k}\right) I C R_{C_{k}}}{\sum_{i=1}^{K} S\left(Q, C_{k}\right)}
$$

Given $I C R_{Q}$, the insulin sensitivity factor of the query case $I S F_{Q}$ is calculated as stated in [27] using Equation (3), where $W$ is the weight of the user in $\mathrm{kg}$.

$$
I S F_{Q}=\frac{341.94 \cdot I C R_{Q}}{W}
$$

After $I C R_{Q}$ and $I S F_{Q}$ are calculated, the recommended bolus $B_{Q}$ for the query case is calculated according to Equation (4).

$$
B_{Q}=\frac{C H O}{I C R_{Q}}+\frac{G_{c}-G_{s p}}{I S F_{Q}}-I O B
$$

This expression is used in bolus calculators with the same input data (see [14]). The remaining active insulin $I O B$ can be calculated as in [15].

\section{E. Revise}

The outcome of the proposed solution can be evaluated by the postprandial BG curve of the user. The proposed revise process is based on the idea proposed in [15], which relies on the assumption that an additional bolus would have been necessary to bring the BG curve in the glycaemic target range in the event that it is not. The authors propose focusing on the minimum postprandial BG value, $G_{m i n}$, to calculate the additional bolus. Given the meal time $t_{m}$, the value $G_{m i n}$ is calculated as expressed by Equation (5) as the minimum BG value of $G_{c g m}(t)$ measured by a CGM between $t_{1}$ and $t_{2}$ time after $t_{m}$, with $t_{1}<t_{2}$, e.g. $t_{1}=2 \mathrm{~h}$ and $t_{2}=6 \mathrm{~h}$.

$$
G_{\text {min }}=\min _{t \in\left\{t_{m}+t_{1}, t_{m}+t_{2}\right\}}\left\{G_{c g m}(t)\right\}
$$

Given $G_{\text {min }}$, this paper proposes to correct the $I C R_{Q}$ recommended by the reuse process according to Equation (6), where $I C R_{Q}$ is the corrected ICR of the query case, and $\alpha \in[0,1]$ is the learning rate. The ISF is also corrected using Equation (3) but with the new $I C \hat{C R}_{Q}$. Note that a learning rate is incorporated to smooth changes since they could be influenced by noisy measurements of the continuous glucose monitor.

$$
I \hat{C R_{Q}}=(1-\alpha) I C R_{Q}+\alpha \frac{C H O+\frac{G_{c}-G_{s p}}{341.94 / W}}{B_{Q}+I O B+\frac{G_{m i n}-G_{l}}{I S F_{Q}}}
$$

Note that the correction bolus is estimated by comparing the postprandial minimum blood glucose $G_{\min }$ and the lower glucose threshold $G_{l}$. Therefore, the revise step modifies the ICR to bring the $G_{m i n}$ to $G_{l}$. The target glucose values, $G_{s p}$ and $G_{l}$, may be agreed upon between the patient and the clinician.

\section{F. Retain}

The retain step is responsible for updating the case base for further recommendations. Hence, there is a maintenance process consisting of deciding if the query case should be saved in the case base, and which cases should be removed from the case base because they are redundant, old, etc.

In the posed problem, the ICR can change over time because subject's physiology changes due to age, changes in body weight, etc. This change cannot be solved by retrieving past experiences since the subject's physiology is new. PepperRec deals with this problem, called concept drift, in this stage. Thus, the proposed maintenance strategy consists of preferring to keep recent cases (i.e. the query case) over similar cases $C_{i}$ in the case base if they are sufficiently similar, i.e. if $S\left(Q, C_{i}\right)<\Sigma$, where $\Sigma$ is a similarity threshold, case $C_{i}$ is removed. This maintenance process relies on the assumption that similar cases should have the same (or very similar) ICR and, if not, the case should be removed because subject's physiology has changed. Insulin response is quite sensitive to input factors of the query case. Therefore, this strategy emphasizes factors interaction in the final recommendation over other cases frequently applied. Then, similar old cases should always be removed because they are either redundant (have the same ICR) or obsolete.

\section{G. Experimentation Set-up}

The proposed CBR-based bolus recommender system has been tested on 33 in silico subjects (11 adults, 11 adolescents and 11 children) using the UVA/PADOVA T1DM simulator [28]. The UVA/PADOVA simulator is the only T1DM simulator approved by the United States Food and Drug Administration as an alternative to animal testing of T1DM control strategies. The 33 virtual subjects are supposed to encompass variations in the population with T1DM [29]. However, since the simulator does not incorporate intra-day variability of insulin sensitivity, and physical activity variability of the ICR, these were artificially introduced as proposed in [30].

Fifty 90-day simulations have been carried out for all 33 virtual subjects. Meal size estimations are done following a random uniform distribution of $[0.7,1.1]$ times the actual meal 
size, and BG readings are taken using the default CGM of the simulator with the default measurement noise, which follows a Johnson's $S_{U}$-distribution with $\gamma=-0.5444, \lambda=10.63827$, $\delta=1.6898$ and $\xi=-5.47$. The learning rate of the revise step has been set to alpha $=0.2$.

The proposed bolus recommender system is analysed in terms of time in, above and under the target glycaemic range, i.e. the proportion of time (in \%) that each subject has the $\mathrm{BG}$ in, below and above the range $[70,180] \mathrm{mg} / \mathrm{dl}$. Wilcoxon tests have been used to verify if the results obtained by PepperRec present significant differences to other methods: the state-ofthe-art method presented in [15], the R2R algorithm explained in [16] and the default bolus therapy provided by the simulator as a baseline method for optimising bolus calculators. Wilcoxon tests are non-parametric statistical hypothesis tests that assess if samples come from continuous distributions with equal medians. This test was chosen because it does not require the assumption that the analysed data follows a normal distribution.

\section{RESULTS}

\section{A. Overall Performance}

Table II shows the results (average \pm standard deviation) of the time $(\%)$ in, below and above the glycaemic target using the proposed method (PepperRec), the R2R method explained in [16], the default insulin therapy of the simulator, and the method in [15] labelled as Herrero et al. The results show that PepperRec achieves a greater amount of time in the glycaemic target range by reducing the time below the target. R2R and Herrero et al.'s methods achieve similar results to the default insulin therapy for adults and adolescents, but worse results for children, who are the ones with higher variability. However, PepperRec outperforms the default insulin therapy even with children and reduces the standard deviation in comparison with the other methods, meaning that it increases the stability of a subject's glucose level. Therefore, PepperRec can recommend a more accurate bolus dosage.

Figures 3, 4 and 5 illustrate the individualised time in, below and above the glycaemic target range for the 11 virtual adults, showing that PepperRec achieves greater amounts of time in the glycaemic target range than the other tested methods for all subjects. Wilcoxon tests show that PepperRec not only significantly $\left(p_{\text {value }}<0.05\right)$ outperforms the other methods in average in terms of time in the glycaemic target, but also outperforms them for all virtual subjects individually (adults, adolescents and children).

\section{B. Robustness}

Figure 6 shows the target in the glycaemic range of the virtual subjects using PepperRec with and without missing values. The results show that when values are missing $(10 \%$ of physical activity is missing) this slightly reduces the time in the glycaemic range. Despite this small performance decrease, the achieved time in the glycaemic range is significantly better than that achieved by the other methods without missing values (see Figure 3), indicating the robustness of PepperRec against missing values. These results are achieved under conditions

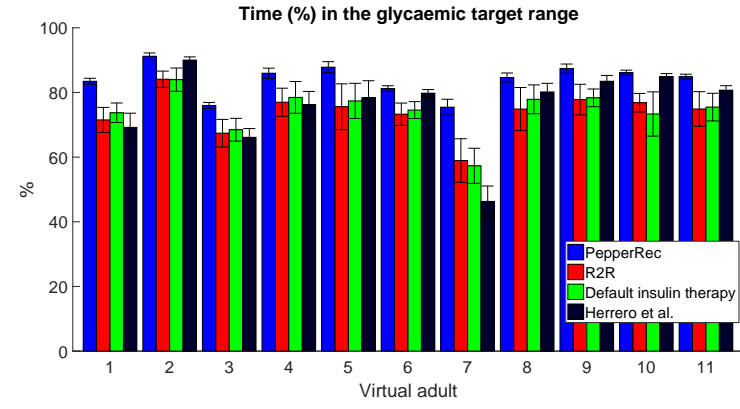

Fig. 3. Average and standard deviation of time (\%) in the glycaemic range of the CBR recommender system PepperRec and a run-to-run recommender system.

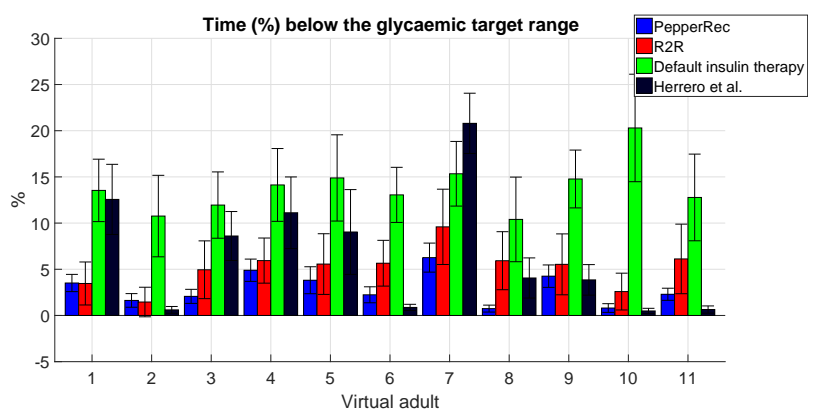

Fig. 4. Time (\%) below the glycaemic range of the CBR recommender system PepperRec and a run-to-run recommender system.

involving the use of a noisy CGM, and mis-estimations of meal size by the subjects, as described in Section II-G.

Basal insulin is the cornerstone of insulin therapy. Table III shows a comparison of the results achieved with PepperRec with the optimal basal dosage and the default basal dosage in terms of the ages of the population under consideration (adults, adolescents and children). The results show that a good basal dosage (the simulators default basal dosage) but not optimal, can reduce the potential benefits of using PepperRec, demonstrating that basal insulin is crucial. In particular, an average absolute deviation about $9 \%$ regarding the optimal basal in adult produces a $5 \%$ reduction of the time in the glycaemic target range; a deviation about $15 \%$ produces a reduction of about $3 \%$ in the portion of time in the glycaemic target range; and a deviation about $55 \%$ produces a reduction

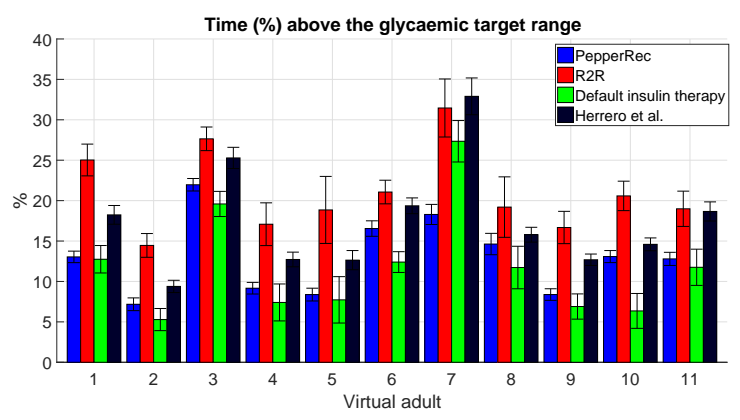

Fig. 5. Time (\%) above the glycaemic range of the CBR recommender system PepperRec and a run-to-run recommender system. 
TABLE II

AVERAGE THROUGHOUt THE 11 ADULTS, 11 ADOLESCENTS AND 11 CHILDREN OF THE MEAN AND STANDARD DEVIATION OF THE TIME (\%) IN, BELOW AND ABOVE THE GLYCAEMIC TARGET USING THE DEFAULT INSULIN THERAPY, A RUN-TO-RUN ALGORITHM TO ADJUST BOLUS CALCULATOR PARAMETERS (R2R), THE METHOD PRESENTED IN [15] (HERRERO ET AL.) AND PEPPERREC. All METHODS HAVE BEEN TESTED USING EMPIRICALLY OPTIMISED CONSTANT BASAL DOSAGE.

\begin{tabular}{|c|c|c|c|c|c|c|c|c|c|}
\hline \multirow[t]{2}{*}{ Method/Subject } & \multicolumn{3}{|c|}{ Adults } & \multicolumn{3}{|c|}{ Adolescents } & \multicolumn{3}{|c|}{ Children } \\
\hline & In & Below & Above & In & Below & Above & In & Below & Above \\
\hline PepperRec with optimal basal & $84.00 \pm 1.26$ & $2.96 \pm 0.93$ & $13.04 \pm 0.87$ & $67.83 \pm 2.07$ & $4.84 \pm 1.75$ & $27.34 \pm 1.09$ & $64.31 \pm 2.21$ & $7.30 \pm 2.10$ & $28.39 \pm 1.15$ \\
\hline R2R & $73.83 \pm 4.72$ & $5.16 \pm 2.87$ & $21.00 \pm 2.41$ & $54.18 \pm 6.39$ & $7.98 \pm 4.58$ & $37.84 \pm 2.47$ & $44.48 \pm 3.71$ & $4.83 \pm 2.94$ & $50.69 \pm 1.77$ \\
\hline Default insulin therapy & $74.44 \pm 4.26$ & $13.81 \pm 4.06$ & $11.75 \pm 2.02$ & $55.21 \pm 9.12$ & $18.28 \pm 8.97$ & $26.51 \pm 2.38$ & $54.11 \pm 6.33$ & $14.86 \pm 5.43$ & $31.04 \pm 2.08$ \\
\hline Herrero et al. & $75.92 \pm 2.75$ & $6.60 \pm 2.13$ & $17.48 \pm 1.11$ & $49.34 \pm 5.53$ & $9.68 \pm 4.54$ & $40.98 \pm 2.37$ & $48.57 \pm 4.10$ & $17.74 \pm 3.47$ & $33.68 \pm 1.75$ \\
\hline
\end{tabular}

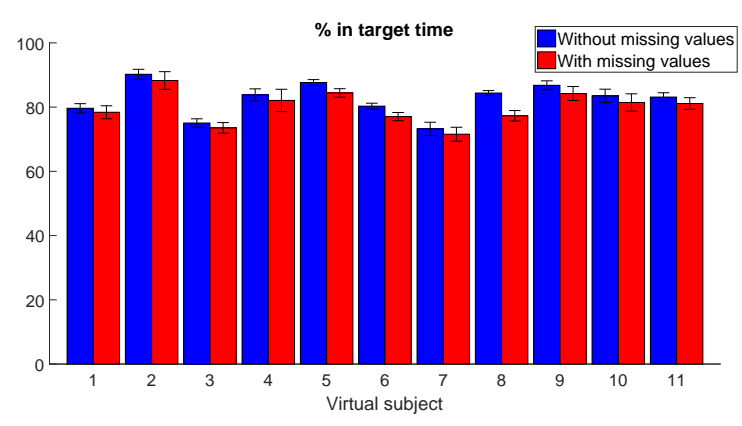

Fig. 6. Time (\%) in the the glycaemic range of PepperRec with and without missing values.

about $11 \%$ in the portion of time inside the target range. Therefore, PepperRec needs a good basal dosage to achieve the best possible results. Nevertheless, PepperRec achieves good results even when basal dosage is far from optimal.

\section{Latency}

PepperRec is capable of recommending bolus doses using experiences stored in the case base. The case base is personalised since it contains the user's experiences. This implies that the first time a bolus is required with regard to a particular context, PepperRec would provide a recommendation based on other experiences in other contexts that may not be sufficiently similar. In such a situation, the recommendation may not be optimal. This problem occurs when new contexts appear, and therefore PepperRec needs to update (or initialise) the case base for the new context.

Figure 7 shows the evolution of the time (average and standard deviation) in the glycaemic target range for all virtual adults in the first 30 days, starting with a case base with a single case with non-optimal ICR and ISF. It shows that the time in the glycaemic target range increases over the days, and finally converges around a value after two weeks. The convergence time can be adjusted with the learning rate of the revise step. However, there is a trade-off between the convergence time and the variability around the optimal after convergence.

\section{DISCUSSION}

The results achieved by PepperRec demonstrate that the variability of the ICR due to factors such as exercise or intraday variability are caught by the proposed system. Thus, the proposed system divides the solution space according to the information provided, and then adapts and personalises the

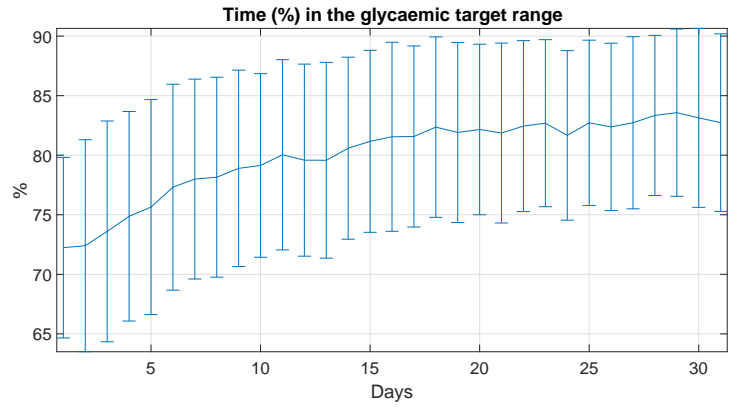

Fig. 7. Evolution of the average and standard deviation of the time (\%) in the the glycaemic range between all virtual adults throughout the first 30 days.

proposed solution. The retain step divides the solution space by keeping the relevant cases however many there are. These cases are then used by the retrieve and reuse steps to propose solutions to new cases. Finally, the revise and retain steps are responsible for repeatedly optimising the proposed solutions and keeping the system updated. Using this methodology, the virtual subjects achieve a time in the glycaemic range that is about $10 \%$ greater than when using the default insulin therapy of the simulator. PepperRec also reduces the variability of the simulations. Therefore, it is capable of improving the safety of patients not only by increasing the time in the target range, but also by increasing the stability of the BG level against different sources of variability.

PepperRec outperforms other methods in the literature such as the one presented in [15] and the R2R algorithm explained in [16] that achieve results similar to those in the default insulin therapy. The CBR bolus recommender system proposed in [14] also achieves similar results to the default insulin therapy as the author explains in [14]. Therefore, PepperRec outperforms these other state-of-the-art methods.

PepperRec is also capable of dealing with missing values and retrieving the most similar experiences in order to recommend a new bolus dose prioritising the most complete cases in the case base. This procedure is proven to be robust when information is missing information by obtaining similar times in the glycaemic range to those when all the information is known. Consequently, PepperRec can minimise a decrease in performance in the event of a loss of information. This conclusion is relevant for healthcare applications, since the system must be trustworthy, even when the user does not provide all the information required due to omission or tiredness.

As with any CBR system, PepperRec requires a good case base to achieve notable results. The research shows that 
TABLE III

AVERAGE THROUgHOUt THE 11 ADULTS, 11 ADOLESCENTS AND 11 CHILDREN OF THE MEAN AND STANDARD DEVIATION OF THE TIME (\%) IN, BELOW AND ABOVE THE GLYCAEMIC TARGET USING PEPPERREC WITH OPTIMISED BASAL DOSAGE USING THE ALGORITHM OF [31] AND THE DEFAULT BASAL DOSAGE OF THE SIMULATOR. THE LAST ROW INDICATES THE AVERAGE AND STANDARD DEVIATION AMONGST SUBJECTS OF THE RELATIVE ABSOLUTE DIFFERENCE BETWEEN THE OPTIMAL BASAL AND DEFAULT BASAL.

\begin{tabular}{|c|c|c|c|c|c|c|c|c|c|}
\hline Method/Subject & \multicolumn{3}{|c|}{ Adults } & \multicolumn{3}{|c|}{ Adolescents } & \multicolumn{1}{c|}{ Children } \\
\hline & In & Below & Above & In & Below & Above & In & Below & Above \\
\hline PepperRec with optimal basal & $84.00 \pm 1.26$ & $2.96 \pm 0.93$ & $13.04 \pm 0.87$ & $67.83 \pm 2.07$ & $4.84 \pm 1.75$ & $27.34 \pm 1.09$ & $64.31 \pm 2.21$ & $7.30 \pm 2.10$ & $28.39 \pm 1.15$ \\
\hline PepperRec with default basal & $79.06 \pm 2.64$ & $6.81 \pm 2.74$ & $14.12 \pm 1.48$ & $64.43 \pm 3.48$ & $8.48 \pm 4.14$ & $27.08 \pm 1.64$ & $53.84 \pm 3.47$ & $16.17 \pm 3.90$ & $30.52 \pm 1.25$ \\
\hline Basal insulin relative difference (\%) & \multicolumn{4}{|c|}{$8.90 \pm 7.47$} & \multicolumn{5}{|c|}{$15.44 \pm 10.94$} \\
\hline
\end{tabular}

PepperRec may need two weeks to initialise the case base, which is an acceptable amount of time since diabetes is a chronic disease, but more research could be done to reduce the effects of the initialisation problem.

\section{CONCLUSIONS}

This paper presents PepperRec, a case based reasoning bolus recommender system for people with type 1 diabetes mellitus. The proposed system automatically estimates the personalised insulin to carbohydrates ratio and insulin sensitivity factor of the user to calculate the appropriate bolus for a given time of day, carbohydrate intake, physical activity, blood glucose level, weight, the desired glucose target and glucose lower bound. Using CBR methodology, PepperRec provides a new retrieve method that is able to deal with missing values. In the reuse phase, PepperRec can manage contextual information due to an adaptation method that combines previous ICR values from similar circumstances. The proposed solution is then corrected according to the outcome (postprandial blood glucose) in the revise phase. Finally, the proposed methodology can repeatedly follow a subject's physiological changes by means of a retain strategy that manages the concept drift by removing obsolete cases in favour of recent ones.

The system has been tested using the UVA/PADOVA simulator with 33 virtual subjects (11 adults, 11 adolescents and 11 children) with artificially-added variability for the time of day and physical activity. The results are compared with the default insulin therapy of the simulator and other methods described in the literature, and demonstrate that the proposed system outperforms the other methods, while exhibiting a robust behaviour when information is missing.

For future work, the system should be tested in a real environment with real people with type 1 diabetes, and the true benefits of the proposed system should be analysed. It would be interesting to analyse the capacity of the proposed methodology to recommend bolus doses to prevent hyperglycaemia when this is forecast and there is still research to be done to minimise the effects of the problem of initialisation.

\section{ACKNOWLEDGMENTS}

This project has received funding from the grant of the University of Girona 2016-2018 (MPCUdG2016) and the European Union Horizon 2020 research and innovation programme under grant agreement No. 689810, www.pepper.eu.com/, PEPPER. The work has been developed with the support of the research group SITES awarded with distinction by the Generalitat de Catalunya (SGR 2014-2016).
Members of the PEPPER Group are as follows: project leader: C. Martin; project management team: D. Brown, F. Torrent-Fontbona, P. Herrero Vinas, L. Nita, J. Masoud, J.M. Fernández-Real, B. López; co-investigators: A. Aldea, D. Duce, M. Fernández-Balsells, P. Gay, P. Georgiou, R. Harrison, B. Innocenti, Y.Leal, N. Oliver, R. Petite, M. Reddy, C. Roman, J. Shapley, M. Waite and M. Wos.

\section{REFERENCES}

[1] World Health Organization, "Global Report on Diabetes," World Health Organization, Tech. Rep., 2016. [Online]. Available: http: //www.who.int/diabetes/global-report/en/

[2] International Diabetes Federation, "2015 Diabetes Atlas," International Diabetes Federation, Tech. Rep., 2015. [Online]. Available: http: //www.diabetesatlas.org/resources/2015-atlas.html

[3] Diabetes Control and Complications Trial Research Group, "The effect of intensive treatment of diabetes on the development and progression of long-term complications in insulin-dependent diabetes mellitus," The New England Journal of Medicine, vol. 329, no. 14 pp. 977-986, 1993. [Online]. Available: http://www.nejm.org/doi/pdf/ 10.1056/NEJM199309303291401

[4] S. Schmidt and K. Nørgaard, "Bolus Calculators," Journal of Diabetes Science and Technology, vol. 8, no. 5, pp. 1035-1041, sep 2014.

[5] S. K. Garg, T. R. Bookout, K. K. McFann, W. C. Kelly, C. Beatson, S. L. Ellis, R. S. Gutin, and P. A. Gottlieb, "Improved Glycemic Control in Intensively Treated Adult Subjects with Type 1 Diabetes Using Insulin Guidance Software," Diabetes Technology \& Therapeutics, vol. 10, no. 5, pp. 369-375, oct 2008.

[6] A. Sussman, E. J. Taylor, M. Patel, J. Ward, S. Alva, A. Lawrence, and R. Ng, "Performance of a Glucose Meter with a Built-in Automated Bolus Calculator versus Manual Bolus Calculation in Insulin-Using Subjects," Journal of Diabetes Science and Technology, vol. 6, no. 2, pp. 339-344, mar 2012

[7] F. J. Doyle, L. M. Huyett, J. B. Lee, H. C. Zisser, and E. Dassau, "Closed-Loop Artificial Pancreas Systems: Engineering the Algorithms," Diabetes Care, vol. 37, no. 5, pp. 1191-1197, may 2014.

[8] G. Lepore, A. Dodesini, I. Nosari, C. Scaranna, A. Corsi, and R. Trevisan, "Bolus calculator improves long-term metabolic control and reduces glucose variability in pump-treated patients with Type 1 diabetes," Nutrition, Metabolism and Cardiovascular Diseases, vol. 22, no. 8, pp. e15-e16, 2012.

[9] B. Shashaj, E. Busetto, and N. Sulli, "Benefits of a bolus calculator in pre- and postprandial glycaemic control and meal flexibility of paediatric patients using continuous subcutaneous insulin infusion (CSII)," Diabetic Medicine, vol. 25, no. 9, pp. 1036-1042, aug 2008.

[10] T. M. Gross, D. Kayne, A. King, C. Rother, and S. Juth, "A Bolus Calculator Is an Effective Means of Controlling Postprandial Glycemia in Patients on Insulin Pump Therapy," Diabetes Technology \& Therapeutics, vol. 5, no. 3, pp. 365-369, jun 2003.

[11] A. Aamodt and E. Plaza, "Case-based reasoning: Foundational issues, methodological variations, and system approaches," AI communications, vol. 7, no. 1, pp. 39-59, 1994

[12] G. Widmer and M. Kubat, "Learning in the presence of concept drift and hidden contexts," Machine Learning, vol. 23, no. 1, pp. 69-101, apr 1996. [Online]. Available: http://link.springer.com/10.1007/BF00116900

[13] C. Poerschke, "Development and evaluation of an intelligent handheld insulin dose advisor for patients with Type 1 diabetes," Ph.D. dissertation, Oxford Brookes University, 2004. [Online]. Available: http://ethos.bl.uk/OrderDetails.do?uin=uk.bl.ethos .401010

[14] D. Brown, "Temporal case-based reasoning for insulin decision support," Ph.D. dissertation, Oxford Brookes University, 2015. 
[15] P. Herrero, P. Pesl, J. Bondia, M. Reddy, N. Oliver, P. Georgiou, and C. Toumazou, "Method for automatic adjustment of an insulin bolus calculator: In silico robustness evaluation under intra-day variability," Computer Methods and Programs in Biomedicine, vol. 119, no. 1, pp. $1-8$, apr 2015.

[16] P. Herrero, P. Pesl, M. Reddy, N. Oliver, P. Georgiou, and S. Member, "Advanced Insulin Bolus Advisor based on Run-To-Run Control and Case-Based Reasoning," IEEE Journal of Biomedical and Health Informatics, vol. 19, no. 3, pp. 1087-1096, 2015.

[17] S.-W. Liu, H.-P. Huang, C.-H. Lin, and I.-L. Chien, "Fuzzy-Logic-Based Supervisor of Insulin Bolus Delivery for Patients with Type 1 Diabetes Mellitus," Industrial \& Engineering Chemistry Research, vol. 52, no. 4, pp. 1678-1690, jan 2013.

[18] C. C. Palerm, H. Zisser, W. C. Bevier, L. Jovanovic, and F. J. Doyle, "Prandial Insulin Dosing Using Run-to-Run Control: Application of clinical data and medical expertise to define a suitable performance metric," Diabetes Care, vol. 30, no. 5, pp. 1131-1136, may 2007.

[19] F. M. Léon Vargas, "Design and implementation of a closed-loop blood glucose control system in patients with type 1 diabetes," $\mathrm{Ph} . \mathrm{D}$. dissertation, University of Girona, 2013. [Online]. Available: http://dugi-doc.udg.edu/handle/10256/8550

[20] A. Rodriguez Herrero, "Propuesta De Algoritmo De Control En Lazo Cerrado De La Diabetes Tipo 1," Ph.D. dissertation, Universidad Politecnica de Madrid, 2010. [Online]. Available: http://oa.upm.es/ 3681/1/AGUSTIN \{_\}RODRIGUEZ\{_\}HERRERO.pdf

[21] R. Hovorka, "Closed-loop insulin delivery: from bench to clinical practice," Nature Reviews Endocrinology, vol. 7, no. 7, pp. 385-395, feb 2011.

[22] R. Lopez de Mantaras, D. McSherry, D. Bridge, D. Leake, B. Smyth, S. Craw, B. Faltings, M. L. Maher, M. T. Cox, K. Forbus, M. Keane, A. Aamodt, and I. Watson, "Retrieval, reuse, revision and retention in case-based reasoning," The Knowledge Engineering Review, vol. 20, no. 03 , p. 215,2005 .

[23] K. J. Cios and G. W. Moore, "Uniqueness of medical data mining," Artificial intelligence in medicine, vol. 26, no. 1, pp. 1-24, 2002.

[24] D. Roqueiro, M. J. Witteveen, V. Anttila, G. M. Terwindt, A. M J. M. van den Maagdenberg, and K. Borgwardt, "In silico phenotyping via co-training for improved phenotype prediction from genotype." Bioinformatics (Oxford, England), vol. 31, no. 12, pp. i303-10, 2015.

[25] A. Tversky, "Features of similarity." Psychological Review, vol. 84, no. 4, pp. 327-352, 1977

[26] C. Pous, P. Gay, A. Pla, J. Brunet, J. Sanz, T. R. y. Cajal, and B. López, "Modeling Reuse on Case-Based Reasoning with Application to Breast Cancer Diagnosis," in Artificial Intelligence: Methodology, Systems, and Applications. Springer Berlin Heidelberg, 2008, pp. 322-332.

[27] J. Walsh, R. Roberts, and T. Bailey, "Guidelines for Optimal Bolus Calculator Settings in Adults," Journal of Diabetes Science and Technology, vol. 5, no. 1, pp. 129-135, jan 2011
[28] C. D. Man, F. Micheletto, D. Lv, M. Breton, B. Kovatchev, and C. Cobelli, "The UVA/PADOVA Type 1 Diabetes Simulator: New Features," Journal of Diabetes Science and Technology, vol. 8, no. 1, pp. 26-34, jan 2014.

[29] R. Visentin, C. Dalla Man, B. Kovatchev, and C. Cobelli, "The University of Virginia/Padova Type 1 Diabetes Simulator Matches the Glucose Traces of a Clinical Trial," Diabetes Technology \& Therapeutics, vol. 16, no. 7, pp. 428-434, jul 2014.

[30] P. Herrero, J. Bondia, O. Adewuyi, P. Pesl, M. El-Sharkawy, M. Reddy, C. Toumazou, N. Oliver, and P. Georgiou, "Enhancing automatic closedloop glucose control in type 1 diabetes with an adaptive meal bolus calculator in silico evaluation under intra-day variability," Computer Methods and Programs in Biomedicine, vol. 146, pp. 125-131, jul 2017.

[31] F. Torrent-Fontbona, "Adaptive basal insulin recommender system based on Kalman filter for type 1 diabetes," Expert Systems with Applications, vol. 101, pp. 1-7, 2018.

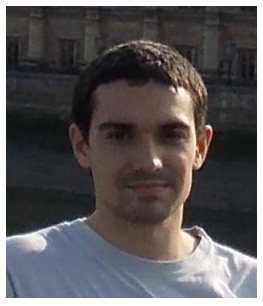

Ferran Torrent-Fontbona received the Electrical and Electronic Engineering degree (2011) from the Polytechnique University of Catalonia (UPC), the M.Sc. in Artificial Intelligence (2012) from the University of Girona and the Ph.D. degree (2015) in the Department of Electrical, Electronic Engineering and Automation of University of Girona.

$\mathrm{He}$ is currently a research associate of the eXiT research group of the University of Girona. He has worked as Professor Assistant and Research in Newronia S.L. Assistant at the UPC, and as Software Developer

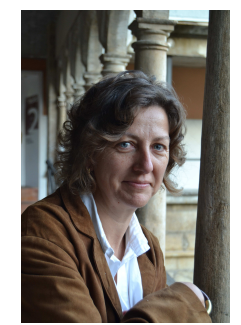

Beatriz Lopez is graduated in Computer Science from the Autonomous University of Barcelona in 1986. Two years later, she joined the Artificial Intelligence Research Group of the Spanish Scientific Research Council where she received her $\mathrm{PhD}$ in Computer Science from the Technical University of Catalonia in 1993.

Since 2000, she is a senior lecturer at the University of Girona. Previous she served at the Rovira Virgili University, and several private companies. In February 2011, she (co)founded Newronia, a company specialised in enterprise resource optimisation. 